\title{
Group-type separation and simulated distillation: a niche for SFC
}

\author{
D.R.P. Thiébaut ${ }^{1}$ and E.C. Robert ${ }^{2}$
}

${ }^{1}$ Laboratoire Environnement et Chimie Analytique (Unité Associée au CNRS), École Supérieure de Physique et de Chimie Industrielles de la Ville de Paris, 10 rue Vauquelin, 75231 Paris Cedex 5, France

${ }^{2}$ Institut Français du Pétrole, BP 311, F-92506 Rueil Malmaison Cedex, France

Recent advances in supercritical-fluid chromatography (SFC) of petroleum fractions are reviewed. Simulated distillation (simdis) still requires some improvements of the hardware so that routine analysis of heavy fractions can be performed. Compared to gas-chromatography simdis, SFC simdis extends the range of this application up to $\mathbf{C}_{140}$ hydrocarbons, while multi-detection would allow the differentiation of aromatic and non-aromatic hydrocarbons. SFC has been registered by ASTM for hydrocarbon group-type analysis using carbon dioxide. It enables fast separation of aromatic and non-aromatic fractions, subfractionation of aromatics and easier quantitation than in liquid chromatography. Resins and asphaltenes of heavier samples can be backflushed from the column. The method could be further improved for better resolution and quantitation via multiple detection and more selective stationary and mobile phases $\left(\mathrm{CO}_{2}-\mathrm{SF}_{6}\right.$ mixtures for example).

Keywords: SFC, simulated distillation, group-type separation, petroleum compounds

\section{Introduction}

Application of supercritical-fluid chromatography (SFC) for the separation of petroleum compounds really started in the '80s. SFC combines many advantages of gas chromatography (GC) and liquid chromatography (LC): its efficiency is close to GC, it works with GC detectors and involves mobile phases whose solvating power can be tuned as in LC; thus, SFC fills the gap between GC and LC. Petroleum applications are a niche for SFC because it combines GC detectors to LC-like mobile phase: high molecular-weight compounds that could not elute from a GC column elute in SFC, while universal and sensitive GC detection, such as with the flameionization detector (FID), can still be used. As a consequence, typical SFC applications [1-4] are GC-like separations, e.g. simulated distillation (simdis), and LC-like separations, e.g. hydrocarbon group-separations. However, a GC-like separation does not mean that an open-tubular column must be used. In fact, both packed and capillary columns can be used in SFC, both having their advantages and drawbacks.

Simdis is a routine GC application; however, SFC is very attractive for eluting hydrocarbons having more than 80 carbons, which are difficult to elute in GC or high-temperature GC (HTGC) without cracking, because their elution can be obtained on packed or open-tubular columns at much lower temperature compared to HTGC. As SFC can also elute hydrocarbons starting from $\mathrm{C}_{20}$ to more than $\mathrm{C}_{130}$, it is more a competitor than an alternative to GC.

The most studied type of LC applications in the petroleum industry is hydrocarbon group-type analysis. SFC's best features for this application are the detection capabilities of FID and the properties of carbon dioxide as a mobile phase. Moreover, FID provides easy quantitation of hydro- 
carbon groups because of the similarity of response factors of most hydrocarbons. Replacing a LC by a SFC method is also worth considering to reduce solvent consumption and costs, or for environmental reasons.

This paper will give an overview of the most attractive SFC applications in the petroleum industry: simdis and group-type analysis, as well as trends for future implementation.

\section{Simulated distillation}

Simdis is widely used in the petroleum industry for evaluation of fossil fuels as well as petroleum feeds and cuts treated in refining and conversion processes. Through a calibration curve relating the boiling point of normal paraffins to their elution temperature or retention time, simdis gives the hydrocarbon distribution of the sample (in weight percent) versus the boiling range of the fraction (expressed in Atmospheric Equivalent Boiling Point, AEBP). The conditions used for simdis, the choice of both the column and the experimental conditions [3] are tuned to provide results in agreement with preparative distillation that gives the True Boiling Point (TBP) curve (described in ASTM D2892). Several methods have been standardized (ASTM D2887, D5307) with packed and open-tubular columns for samples with a final boiling point (FBP) up to $538{ }^{\circ} \mathrm{C}\left(1000{ }^{\circ} \mathrm{F}\right)$. Much effort has gone into extending the range of eluted compounds and the standardization of a method up to an FBP of $700{ }^{\circ} \mathrm{C}$ is in progress (ASTM proposed test method, 1994). However, at oven temperatures up to $430{ }^{\circ} \mathrm{C}$ in hightemperature GC, the resistance of high molecular-weight hydrocarbons (HMHs) to cracking reactions is questionable [5] and the use of new thermally-stable columns with metallic clad for routine application of GC simdis in the range of alkanes from $\mathrm{nC}_{5}$ up to $\mathrm{nC}_{72}$ is desirable [6]. The main advantage of SFC over GC techniques comes from the solvent strength of the mobile phase. The polarity of the most commonly used supercritical mobile-phase, $\mathrm{CO}_{2}$, depending on the operating conditions, varies between that of pentane and toluene, making SFC a potentially powerful technique for the elution of $\mathrm{HMH}$ at much lower temperatures than GC. Thus, SFC could be the tool of choice for better quantitation of conversion in heavy petroleum-fraction processing. Another interesting feature of SFC for simdis is the availability of both capillary GC-like columns and LC packed-columns.

\section{Capillary SFC}

Simdis using open-tubular capillary-column SFC (cSFC) is more or less a mimic of GC simdis: it involves similar crosslinked stationary phases in columns of similar geometry as in fast GC. The mobile phase, depending on pressure and temperature, varies from a dense gas to a supercritical fluid. This occurs during the separation of HMHs.
The advantages of capillary columns are based on their better deactivation than packed columns. This is a major benefit for eluting HMHs of any chemical nature, and also for reducing differences between retention times of, for instance, paraffins and aromatics having the same boiling point because a low selectivity is required in simdis.

The drawbacks of cSFC are caused by the miniaturized technique itself: the injection mode requires particular attention due to the low capacity of the column and the introduction of a sample into a high-pressure system compared to GC. In most cases, injection requires splitting of the sample before entering the column, which is a potential source of sample discrimination and of dispersion of the results. Several other approaches have been tested, such as timedsplit injection (several $\mathrm{nl}$ injected) [7] or the use of a retention gap, the latter allowing solutes focusing at the head of the column for trace analysis for example [1]. As in GC, finding a proper solvent for the injection of the sample can be a problem. Carbon disulfide is a good candidate because it gives a low FID response [8-11]. However, it has to be replaced by higher boiling-point solvents, like xylene for injections at elevated temperatures. It is admitted that injection for SFC simdis must be performed at temperature higher than $100{ }^{\circ} \mathrm{C}$ [10-12], mainly for dissolving the standards used for correlating retention times and boiling points.

With regard to the FID, the supercritical fluid has to be depressurized down to atmospheric pressure with the risk of $\mathrm{HMH}$ precipitation and restrictor clogging because of the dramatic decrease of the solvating power of $\mathrm{CO}_{2}$. However, Bouigeon et al. [11] have shown that the behavior of the restrictor was reliable as soon as the final pressure for elution was 55-60 MPa.

Using open-tubular capillary columns, pressure and mobile-phase flow-rate in the column cannot be controlled independently, because only very low dead-volume fixedrestrictors can be used with capillary columns. Generally, syringe pumps are used to deliver the low flow-rate required by the $50-100 \mu \mathrm{m}$ ID capillary columns. However, using a splitter prior to the injection valve, it is possible to use conventional reciprocating pumps and software-controlled automatic pressure-regulation to perform pressure/density gradients in cSFC simdis [13-14]. This mode has been referred as "upstream mode" by Hewlett-Packard, who introduced it in their commercial system (since then Hewlett-Packard has retired from SFC). The system has been modified and commercialized worldwide by Berger Instruments. In fact, the upstream mode can be carried out with any commercial automatic pressure-regulator via a simple modification of the mobile-phase flow-scheme. Because of the large flow-rate going through the pressure regulator, it is necessary to check the flow-rate accuracy in the capillary column, by using either a test separation or by measuring the $\mathrm{CO}_{2}$ gas flowrate in the detector, because the capillary-column flow-rate is negligible versus the splitted flow.

The main results reported in the literature are gathered in table I. In all cases, non-polar stationary-phases are used, including original octyl phases. Very high pressure is 
Table I. Summary of recent capillary SFC-simdis results.

\begin{tabular}{|c|c|c|c|c|c|}
\hline $\begin{array}{l}\text { Authors } \\
\text { (year) [ref.] }\end{array}$ & $\begin{array}{l}\text { Stationary } \\
\text { phase }\end{array}$ & $\begin{array}{c}\text { Column temperature } \\
\left({ }^{\circ} \mathrm{C}\right)\end{array}$ & $\begin{array}{l}\text { Maximum pressure } \\
(\mathrm{MPa})\end{array}$ & $\begin{array}{l}\text { Heaviest alkane } \\
\text { eluted }(B P)\end{array}$ & $\begin{array}{c}\text { Relative deviation } \\
(\%) \text { * }\end{array}$ \\
\hline $\begin{array}{l}\text { Schwartz } \\
\text { (1987) [5] }\end{array}$ & $\begin{array}{l}5 \% \text { phenyl- } \\
\text { methylpolysiloxane }\end{array}$ & 100 & 38.5 & $\mathrm{C}_{80}(675)$ & 0.08 to 9.7 \\
\hline $\begin{array}{l}\text { Raynie } \\
\text { (1991) [7] }\end{array}$ & n-octylpolysiloxane & 150 & 32 & $\mathrm{C}_{100}(719)$ & $\begin{array}{c}0.9 \text { to } 3.2 \\
\left(\mathrm{CO}_{2} \text { at } 100{ }^{\circ} \mathrm{C}\right)\end{array}$ \\
\hline $\begin{array}{l}\text { Shariff } \\
\text { (1994) [15] }\end{array}$ & n-octylpolysiloxane & NA & NA & $\mathrm{C}_{90}(700)$ & 0 to 11 \\
\hline $\begin{array}{l}\text { Bouigeon } \\
\text { (1996) [11] }\end{array}$ & SB-octyl & 180 & 50 & $\mathrm{C}_{96}(712)$ & 8.2 \\
\hline $\begin{array}{l}\text { Bouigeon } \\
\text { (1996) [11] }\end{array}$ & $\begin{array}{c}5 \% \text { phenyl- } \\
\text { methylpolysiloxane }\end{array}$ & 180 & 50 & $C_{92}(704)$ & 11.3 \\
\hline $\begin{array}{l}\text { Bouigeon } \\
\text { (1996) [11] }\end{array}$ & PDMS & 180 & 50 & $\mathrm{C}_{108}(732)$ & 13.5 \\
\hline
\end{tabular}

* Maximum deviation from boiling points obtained by SFC.

NA: non-available data.

required for eluting $\mathrm{HMH}$ exceeding $\mathrm{C}_{100}$ [11] at constant temperature $\left(>100^{\circ} \mathrm{C}\right)$. The density of the mobile phase has to be increased during the separation via pressure programming.

The lowest difference between the retention of different compounds having the same boiling point (e.g. alkanes and aromatics) is needed, meaning there is no selectivity versus structure for the accuracy of distillation curves. All the phases tested by Bouigeon exhibit higher retention for aliphatic hydrocarbons than for aromatic hydrocarbons having the same boiling points, despite the use of a $5 \%$ phenyl stationary-phase [11]. The use of octyl-bonded phase provides a lower deviation of simulated boiling points than that of polydimethylsiloxane and phenyl-methylpolysiloxane stationary-phases compared to the actual boiling points of aromatic hydrocarbons from naphthalene to chrysene $[7,11,15]$.

Up to now, the heaviest compound eluted in cSFC simdis is $\mathrm{C}_{108}$ [11] on polydimethylsiloxane which was found to exhibit lower retention than other tested phases. This result was obtained by increasing both working pressure and temperature.

Dissolving the samples prior to SFC simdis is not trivial. Carbon disulfide is generally used, but, in the standard conditions of temperature and pressure, it cannot dissolve heavy test mixtures such as Polywax 1000 used to correlate retention and boiling points. Xylene is generally preferred, it can be heated up to about $120{ }^{\circ} \mathrm{C}$ to take advantage of better solubilization of the samples, while maintaining the use of a conventional injector [10-12]. Obviously, it can be concluded that the elution limit, $\mathrm{C}_{90}$, reported by previous workers, occured because the final pressure used was too low and, in some cases, because there was discrimination during the sample introduction due to the non availability of a heated injection system.

\section{Packed columns}

Despite the potential of cSFC, most authors turned to packed-column SFC (pSFC) because no split injection is required and the columns provide better loadability. Schwartz [16] first reported on pSFC-simdis using a 1-mm ID column packed with a polysiloxane material. The results were very promising as elution of $\mathrm{nC}_{108}$ was demonstrated from polyethylene PE740 despite the reproducibility of retention times was lower than those achieved with cSFC (Tab. II). However, as this type of stationary phase is no longer commercialized, further developments of SFC simdis were carried out using alkyl-bonded silica stationary phases.

Hewlett Packard [13] used the "downstream mode" for independent control of the $\mathrm{CO}_{2}$ flow rate and column pressure, together with a dual FID and UV detection mode that gives the total hydrocarbon and aromatic profiles at $0.75 \mathrm{ml} / \mathrm{min}$. Pressure was programmed from 10 to $36 \mathrm{MPa}$. The use of the downstream mode was reported to enable better reproducibility than the upstream mode, because the 
Table II. Summary of recent pSFC-simdis results.

\begin{tabular}{|c|c|c|c|c|c|}
\hline $\begin{array}{l}\text { Authors } \\
\text { (year) [ref.] }\end{array}$ & $\begin{array}{l}\text { Stationary } \\
\text { phase }\end{array}$ & $\begin{array}{c}\text { Column temperature } \\
\left({ }^{\circ} \mathrm{C}\right)\end{array}$ & $\begin{array}{l}\text { Maximum pressure } \\
(\mathrm{MPa})\end{array}$ & $\begin{array}{l}\text { Heaviest alkane } \\
\text { eluted (BP) }\end{array}$ & $\begin{array}{c}\text { Relative deviation } \\
(\%)^{*}\end{array}$ \\
\hline $\begin{array}{l}\text { Schwartz } \\
(1988)[16]\end{array}$ & Polysiloxane & 150 & 38.5 & $\mathrm{C}_{100}(719)$ & from 0.18 to 5.4 \\
\hline $\begin{array}{l}\text { Shariff } \\
\text { (1994) [15] }\end{array}$ & $\mathrm{C}_{8}$-bonded silica & 120 & 41.5 & $\mathrm{C}_{130}(777)$ & from 0 to 11 \\
\hline $\begin{array}{l}\text { Shariff } \\
\text { (1994) [15] }\end{array}$ & $\mathrm{C}_{6}$-bonded silica & 120 & 41.5 & $C_{124}(756)$ & from 0 to 8.7 \\
\hline $\begin{array}{l}\text { Sotty } \\
(1994) \text { [10] }\end{array}$ & $\mathrm{C}_{11}$ bonded silica & 180 & $>50$ & $\mathrm{C}_{130}(766)$ & $N A^{* *}$ \\
\hline $\begin{array}{l}\text { Huynh } \\
\text { (1999) [12] }\end{array}$ & $\mathrm{C}_{4}$-bonded silica & 170 & 48 & $\mathrm{C}_{136}(777)$ & $N A^{* *}$ \\
\hline
\end{tabular}

* Maximum deviation from boiling points obtained by SFC.

NA: non available data.

** True Boiling Point is not available for such heavy compounds.

restrictor only controls the mass flow going to the FID, while most of the flow is split towards the UV detector [17]. SFC and HTGC results were reported to be highly consistent. The separation of a test sample (Polywax 655) is shown on figure 1 .

More recent works on pSFC simdis investigated the use of packed-capillary columns (300 $\mu \mathrm{m}$ ID). Sotty et al. [10] used a column packed with an undecyl-bonded silica $\left(\mathrm{C}_{11}\right)$ and eluted the $\mathrm{nC}_{132}$ alkane of Polywax 1000. The authors emphasized the effect of injection temperature (up to $130^{\circ} \mathrm{C}$ ) for elution of the heaviest hydrocarbons. Shariff $e t$ al. [15] observed a retention decrease when decreasing the number of carbon atoms in the alkyl group of the stationary phase, with a slight minimum for the hexyl-bonded silica. They also showed that the deviation between simulated boiling points and actual boiling points of non-alkylated aromatics is minimized using $\mathrm{C}_{8}$-bonded silica.

A very recent work [12] underlines the main parameters for implementing pSFC simdis: it is confirmed that the longer the alkyl chain of the stationary phase, the stronger the retention of hydrocarbons, but no minimum is observed as $\mathrm{C}_{1}$-bonded silica is found to be less retentive than $\mathrm{C}_{4}$. Thus, to reduce the maximum operating pressure, $\mathrm{C}_{1}$-bonded phases should be preferred. For example, an hydrocarbon with 120 carbon atoms $\left(\mathrm{C}_{120}\right)$ is eluted, respectively, at $38 \mathrm{MPa}$ and $42 \mathrm{MPa}$ with Deltabond methyl and Chromasil butyl; increasing the temperature of the column has a major effect on the heaviest compound eluted: on the Chromasil butyl stationary-phase, an operating temperature of $170{ }^{\circ} \mathrm{C}$ enables elution of $\mathrm{C}_{136}$ hydrocarbon (Fig. 2) instead of $\mathrm{C}_{106}$ at $130{ }^{\circ} \mathrm{C}$. As increasing the temperature of the mobile phase leads to a decrease of the $\mathrm{CO}_{2}$ density, maintaining a constant density in the column requires higher operating pressures during the separation; the extended molecular weight of eluted compounds gained by raising the temperature is worth the increase of pressure required for constant density.

Huynh et al. confirm that the lowest difference between the retention of different compounds having the same boiling point is also obtained for bonded phases with long alkyl chains $\left(\mathrm{C}_{8}\right)$, as described by Shariff et al. [15]. A compromise must be made between the following requirements: obtain a low retention (to avoid extreme operating conditions) and maintain selectivity as low as possible. Huynh et al. selected a deactivated C4-bonded silica so that the final operating pressure is less than $50 \mathrm{MPa}$ (still exceeding the capabilities of many reciprocating pumps that can be used in SFC) and the selectivity (expressed as the difference of the boiling points of two co-eluting hydrocarbons) is less than $10{ }^{\circ} \mathrm{C}$.

As supercritical fluids exhibit very good solvent properties, it would be desirable to directly inject heavy fractions containing asphaltenes, thus eliminating the time-consuming and error-prone need to perform sample preparation prior to simdis (removal of asphaltenes is obtained by precipitation with $\mathrm{n}$-heptane). It is unclear if injection of samples containing asphaltenes is realistic in SFC as Peaden [3] mentions their low solubility in supercritical $\mathrm{CO}_{2}$, while Skaar et al. [18] report their injection. Schoenmakers [19] reported precipitation of heavy aromatic compounds. This was not observed by Huynh after injection of oil fractions, but on 


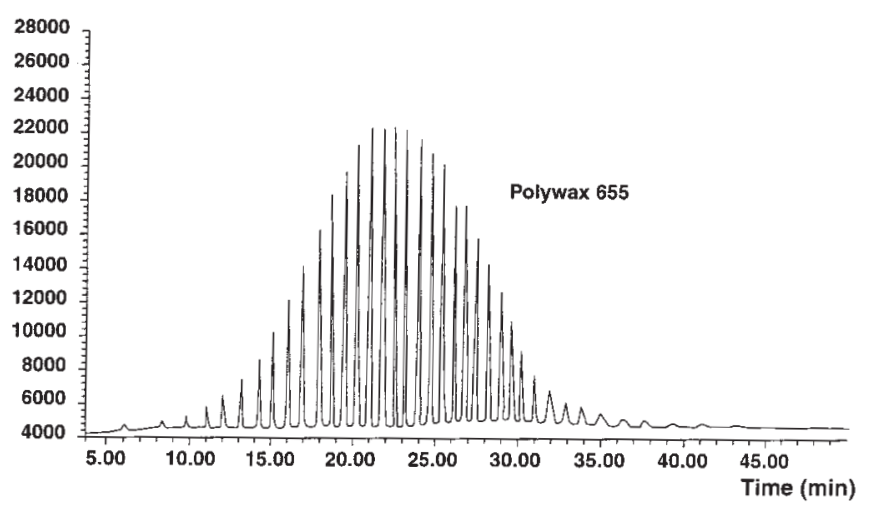

HP SFC packed column chromatograms of polyethylene waxes

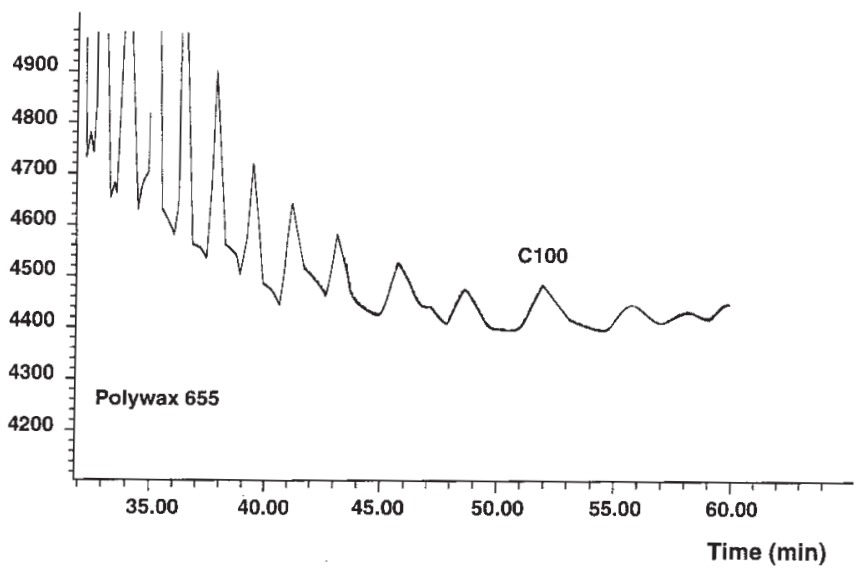

Figure 1. SFC chromatogram of Polywax 655. Conditions: column $200 \times 2.1 \mathrm{~mm}$ Hypersil ODS, $\mathrm{CO}_{2}$ at $150{ }^{\circ} \mathrm{C}$, flow rate of $0.75 \mathrm{ml} / \mathrm{min}$, pressure programming from 10 up to $36 \mathrm{MPa}$, FID at $250{ }^{\circ} \mathrm{C}$ [13].

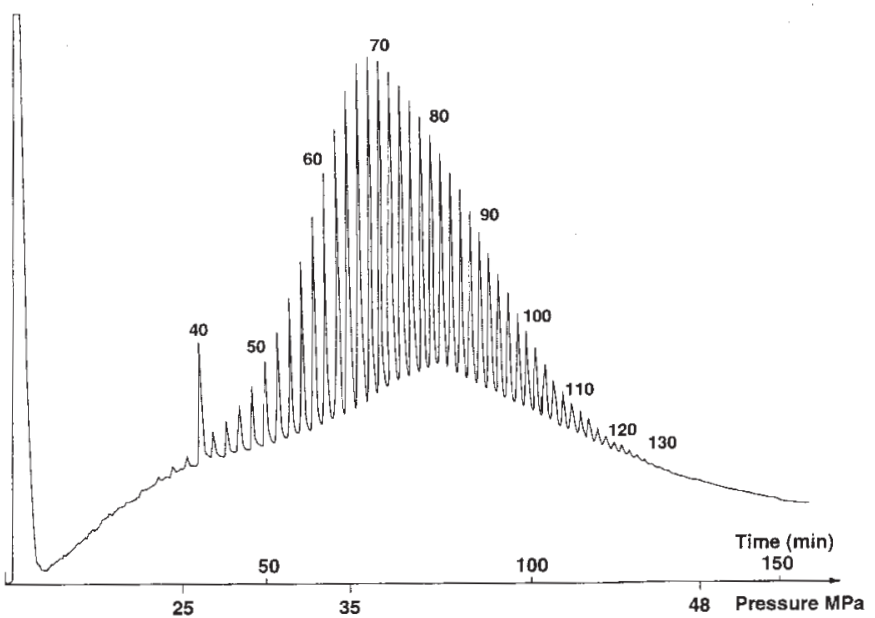

Figure 2. SFC chromatogram of Polywax 1000. Conditions: column $150 \times 0.8 \mathrm{~mm}$ Kromasil $\mathrm{C} 4, \mathrm{CO}_{2}$ at $170{ }^{\circ} \mathrm{C}$, injector temperature $90{ }^{\circ} \mathrm{C}$. FID temperature $420{ }^{\circ} \mathrm{C}$ [12]. calibration standards. Therefore, it is highly recommended to perform a blank injection after the injection of oil or standard samples.

Generally, a high level of consistency is reported between SFC and GC simdis results. Some deviations are reported by Huynh; they come from early-eluting peaks unresolved from the solvent peak, and, for some samples, accuracy of baseline substraction. Huynh reports routine elution of $\mathrm{C}_{120^{-}}$ range hydrocarbons, while the routine GC range is limited to $\mathrm{C}_{80}$. She also demonstrates the validity of the method (Fig. 3 and 4) by comparing the P475-distillate distillation curve with the curve obtained by reconstruction from those of individual hydrocarbon groups obtained by LC fractionation.

Fifteen years of research show the potential of SFC simdis:

- elution of heavier compounds than GC is obtained at much lower temperatures to prevent cracking of the hydrocarbons;

- non-polar LC stationary phases enable elution of hydrocarbons with minimum selectivity; using a non-aqueous mobile phase, such as $\mathrm{CO}_{2}$, they can be used at elevated temperatures without evidence of degradation of their chromatographic performances $[11,12]$ and they are suited to routine analysis.

However, routine SFC-simdis requires an apparatus designed for operation at elevated pressures: all the parts should withstand $60 \mathrm{MPa}$, including the pressure regulator, so that working at about $50 \mathrm{MPa}$ would not be a problem; the design of a new injection system allowing sample introduction at high pressure and temperature is very desirable. A prototype of such an injector has been designed very recently $[20,21]$.

\section{Group-type analysis}

Group-type analysis refers to the separation and quantitation of the hydrocarbon groups of petroleum fractions. This is a difficult analysis because it deals with a large number of very similar molecules, i.e. saturates, olefins, aromatic hydrocarbons and "polar" compounds. Each group type can further be separated into subgroups: saturates into paraffins and cycloalkanes (condensed and non-condensed naphthenes), aromatic hydrocarbons into mono-, di-, tri- and polycyclic aromatic hydrocarbons. These separations are socalled SARA or SOARA (saturates, olefins, aromatics, resins and asphaltenes). A large number of methods are available for the group-type analysis of petroleum distillates such as diesel fuel [22]:

- chromatography (gas, liquid, supercritical);

- mass spectrometry; 


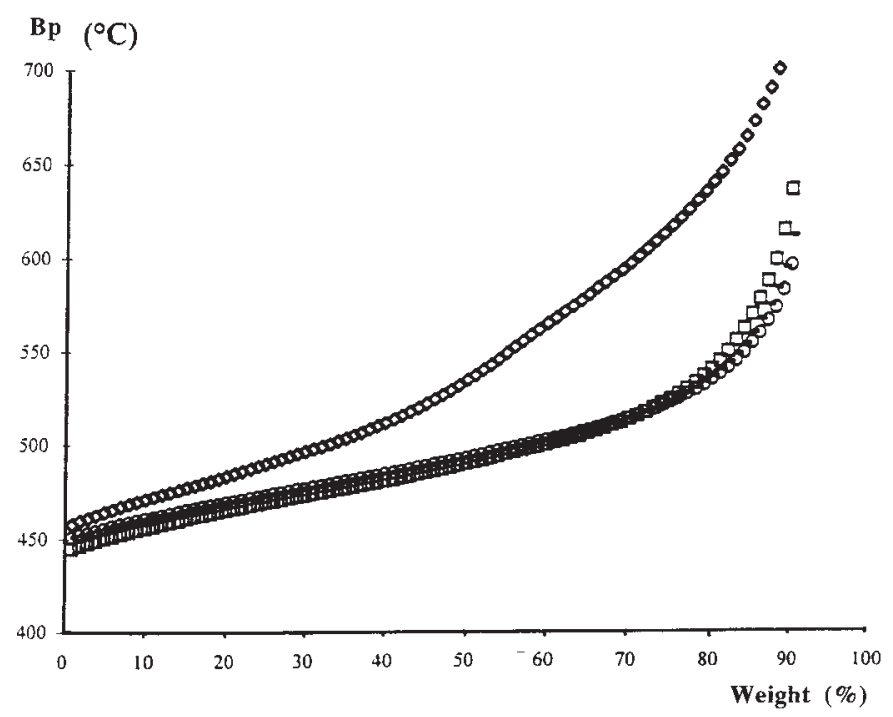

Figure 3. Distillation curves from P475 distillate (0), saturates (-), aromatics $(\square)$ and resins $(\diamond)$ fractions. Conditions: column $150 \times$ $0.8 \mathrm{~mm}$ Kromasil C4, $\mathrm{CO}_{2}$ temperature $150{ }^{\circ} \mathrm{C}$; FID $420{ }^{\circ} \mathrm{C}, \mathrm{CO}_{2}$ gas flow-rate $3.5 \mathrm{ml} / \mathrm{min}$; pressure program: $15 \mathrm{~min}$ at $15 \mathrm{MPa}$, $3 \times 30 \mathrm{~min}$ ramps from 15 to 25 to 32.5 and to $38 \mathrm{MPa}$, and $60 \mathrm{~min}$ ramp to $48 \mathrm{MPa}$.

- spectroscopy, nuclear magnetic resonance (NMR, IP392/90 method), ultraviolet [23] and infrared (IR) or near infrared (NIR).

Depending on the technique, either the carbon type (single or double bond) or the hydrocarbon type (saturate, olefinic or aromatic) is determined. The latter is obtained by chromatography and mass spectrometry. Some mass spectrometric methods were standardized (ASTM D2425, ASTM D3239). They allow the most detailed analysis of hydrocarbon types in middle distillates, but are not suitable for everyday refinery use. Chromatography involves the fluorescentindicator absorption method (ASTM D1319), that is unreliable for distillates with FBP higher than $315^{\circ} \mathrm{C}$ but allows the quantitation of saturates, olefins and aromatics in gasolines and jet fuels [22], thin-layer chromatography (TLC), LC and SFC. LC-GC coupling [24] is also worth mentioning since it provides more detailed information on the composition of the distillates, and benefits, as does SFC, from the flame-ionization detection. SFC-GC has also been reported [25]. At the present time, LC or SFC methods are the most attractive separation techniques for the determination of aromatic group-types. However, LC suffers from refractometric detection calibration, as it has been reported to become inaccurate [26] for some types of processed fuels, in particular, mildly hydrotreated fuels having a higher ratio of (tetraline + indane) / alkylbenzene. For gasolines, a number of GC methods are also described but the detailed analysis obtained by GC (or LC, SFC-GC couplings) are less suited to the determination of total olefinic and aromatic

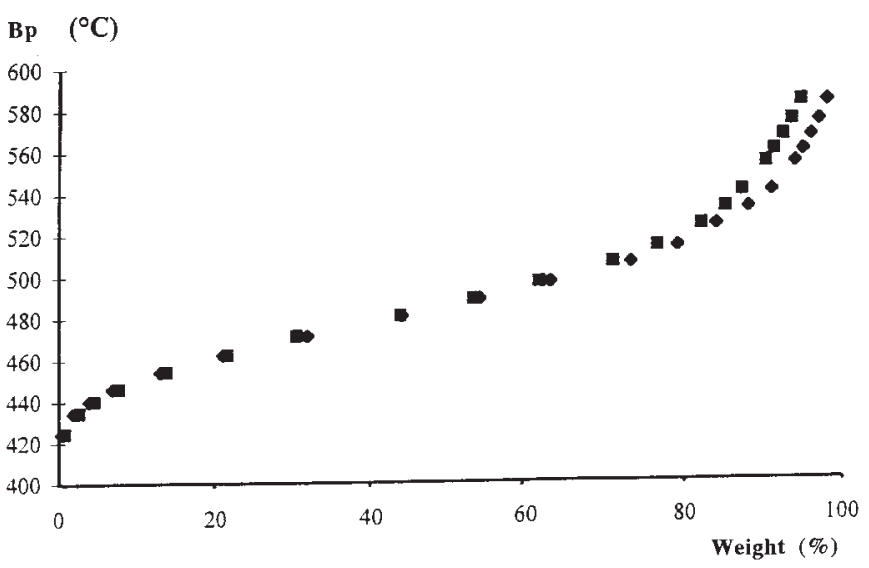

Figure 4. Distillation curves of P475 distillate obtained from crude sample $(\diamond)$ and reconstruction from the fractions curves (ם). Same conditions as figure 3.

contents in commercial gasolines. Thus, SFC is again an alternative technique to be considered.

Separation techniques used for group-type analysis of heavy petroleum fractions (vacuum distillates, residues and crudes) are generally based on the method developed by Snyder [27], i.e. a preparative adsorption-LC step followed by a gravimetric determination of fractions. Deasphalting, for removal of insoluble compounds with n-pentane, n-hexane or n-heptane (asphaltenes) can be necessary and the technique is time-consuming even though miniaturized systems have been proposed [28]. Analytical LC methods have also been proposed and were recently reviewed by Lundanes and Greibrokk [29]; however, they involve complex column switching and backflushing, lack of universal and sensitive detection, and suffer from the composition variability of petroleum fractions.

SFC's main advantage over LC relies on the direct coupling to FID. A number of studies have been published and SFC is registred as ASTM 5186-91 and 5186-96 for the determination of aromatic hydrocarbons in diesel fuels. Some examples are given below.

\section{Middle distillates and gasolines}

\section{Total aromatic content by SFC: ASTM (D5186-91)}

A quantitative study on the determination of total aromatic content in jet fuels and diesel fuels using synthetic mixtures reported by Di Sanzo and Yoder [30] demonstrated the accuracy of SFC and its suitability for an ASTM method. The authors used a $25 \mathrm{~cm} \times 2 \mathrm{~mm}$ ID, $5 \mu \mathrm{m}$ silica column and $\mathrm{CO}_{2}$ at $30^{\circ} \mathrm{C}$ and $11.5 \mathrm{MPa}$ as the mobile phase. The results were very consistent for blends with more than $10 \%(\mathrm{~m} / \mathrm{m})$ of aromatics, but the deviation increased up to $35 \%$ at $3.5 \%(\mathrm{~m} / \mathrm{m})$ total aromatic hydrocarbons because of the lack of resolution between saturate and aromatic hydrocarbons. 
The ASTM method (D5186-91) involves SFC coupled to FID for the determination of total aromatic content of diesel fuels. It requires the selection of a suitable stationary phase so that the resolution between docosane and toluene is at least 4 , in order to provide a satisfactory separation between saturates and aromatics. Fraile and Sanchez [31] used an experimental design approach to optimize analysis time and resolution between saturates and aromatics.

Applications notes from Hewlett Packard [32] and Analytical Control [33] proposed improvements of aromatic determination using the ASTM method via the double detection mode, UV and FID, available on the HP SFC G1205 for a better determination at low aromatic contents. The AC note 9317 reported similar conclusions on the influence of FID conditions as reported by Di Sanzo [30] concerning the deviations observed under optimized and non-optimized conditions.

However, as the method was restricted to total aromatic fraction analysis some extensions have been studied with the aim of adressing subgroup-type determination of aromatics and also of extending the range of SFC to other petroleum cuts, gasoline or heavier distillates.

\section{Hydrocarbon groups by SFC: ASTM method 5186-96}

Various attemps to perform hydrocarbon group-separations by SFC were described before the upgrade of ASTM method 5186. After the first work of Norris et Rawdon [34] showing a satisfactory separation between saturates, olefins and aromatics in gasoline samples using a $20 \%$ silver-loaded silica but lacking reproducibility [35], interesting multidimensional approaches were reported by Campbell et al. [35], Fuhr et al. [36] and Anderson et al. [37, 38]. Campbell et al. [35] separated saturates, olefins and total aromatics in middle distillates using $10 \% \mathrm{~mol} / \mathrm{mol} \mathrm{CO}_{2}$ in $\mathrm{SF}_{6}$ as the mobile phase and two 1-mm-ID columns packed with a silica gel and a silver-loaded strong cation-exchange silica gel. Two experiments were necessary:

- the first involved the silica column for elution of saturates and olefins as a single peak, while aromatics were retained and backflushed off;

- the second experiment used the silver column for elution of aliphatic fraction while $\pi$-donor olefins and aromatics were retained and backflushed off as a single peak.

This difference method leads to a standard deviation of $1.5 \%$ but it could not apply to low-olefinic contents middle distillates. For gasolines, results were satisfactory using the difference method or a column switching using the same columns as the difference method.

Fuhr et al. [36] used a silica column for separation of saturates from aromatics followed by switching of the aromatics on an amino-bonded silica column for subgroup-type determination. Later, Anderson et al. [37-38] got a complete group-type and subgroup-type analysis, including olefins in middle distillates and gasolines, using microcolumns packed with aminopropyl-bonded silica and high specific-area bare silica (two columns in series) for the retention of "polar" fractions and a short silver-loaded silica column for separation of aliphatic and olefinic fractions, unretained on the first two columns (Fig. 5 and 6). The separation between alkanes/alkenes and monoaromatics was reported to be much easier for gasolines because they have a narrower boiling range than middle distillates; however, it was difficult to draw conclusions concerning quantitative analysis as only two low-olefinic gasolines were analysed by SFC and compared to FIA results.

The method was simplified when the ASTM D5186-96 was released; it allows the determination of non-aromatic, monoaromatic and polynuclear aromatic fractions (di+) from diesel and aviation-turbine fuels. In addition to the requirements for resolution between non-aromatic and aromatic fractions, the separation between monoaromatics and polyaromatics is given by the resolution (at least two) between tetraline (eluted in the peak of monoaromatics) and naphtalene. However, at the present time, the SFC method still gives less information than LC since group-type analysis of aromatic compounds is not completely achieved.

Various improvements of the ASTM separation method were proposed with the aim of increasing the resolution
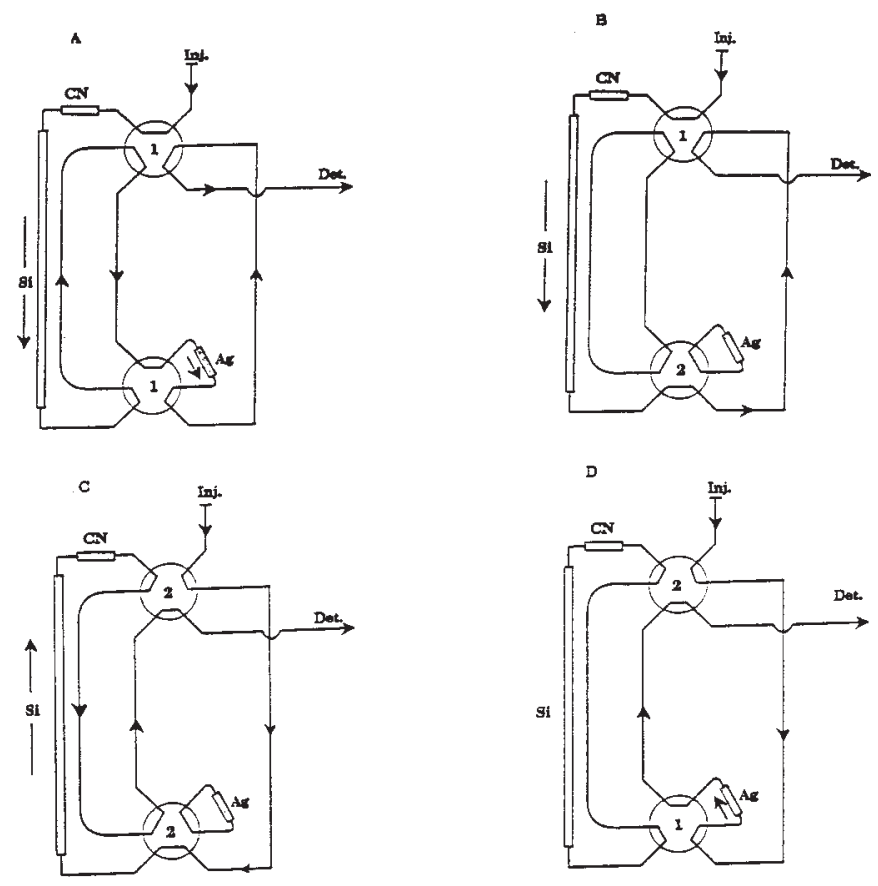

Figure 5. Scheme of multi-dimensional hydrocarbon group-separation SFC-apparatus for diesel fuels analysis [38]. A: retention of polar hydrocarbons on nitrile and bare silica columns; saturates and olefins are trapped in the silver-loaded column. B: elution of aromatics from the silica column and detection. C: backflush of $\mathrm{CN}$ column for detection of polar hydrocarbons and tri- and polyaromatics. A: elution of saturates from the silver-loaded column and detection. D: backflush of olefins from the silver-loaded column for detection. 


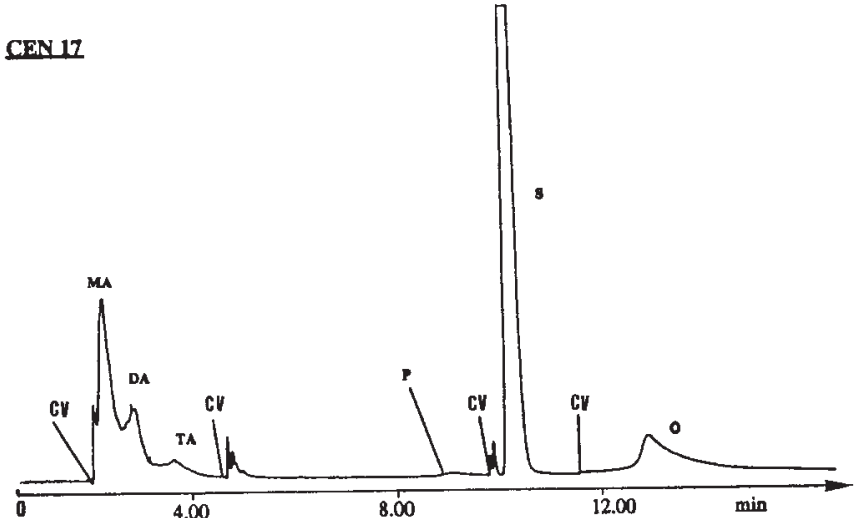

Figure 6. SFC multidimensional chromatogram of CEN 17 hydrocarbon groups [38]. Same conditions as figure $5 . \mathrm{CO}_{2}$ at 37.5 $\mathrm{MPa}$ and $75{ }^{\circ} \mathrm{C}$; CV: change valve-position; S: saturates; MA: monoaromatics; DA: diaromatics; TA: triaromatics; P: polar hydrocarbons; O: olefins.

between the groups for better quantitation or to separate more fractions.

Chen et al. [39] studied aromatic group-type determination on fifty diesel fuels with different refinery streams in the range 6 to $48 \%(\mathrm{~m} / \mathrm{m})$ total aromatics. They used a cyano column connected to a silica column to perform group-type separation with $\mathrm{CO}_{2}$ at $40{ }^{\circ} \mathrm{C}$ and $31.5 \mathrm{MPa}$. The analysis time was less than 45 min. However, it can be concluded from their chromatograms that there is a lack of resolution between saturates and monoaromatics despite the fact that the results obtained by LC, MS (ASTM D2425) and SFC are consistent. The poor resolution between saturates and monoaromatics explains SFC's over-estimation of monoaromatics.

Li et al. [40], in order to extend the scope of the ASTM method to the determination of aromatic subgroups, utilized long capillary columns packed with high specific-area silica. Column lengths of $52 \mathrm{~cm}$ and $98 \mathrm{~cm}$ were used to obtain, respectively, resolutions values of 8.4 and 17 between $\mathrm{nC}_{16}$ and toluene, and 4.7 and 9.3 between tetraline and naphthalene, with $\mathrm{CO}_{2}$ at $15 \mathrm{MPa}$ and $45{ }^{\circ} \mathrm{C}$, while the retention times of naphthalene were reasonable for such column lengths (6 min and 16 min respectively). Similar results with a $1.3 \mathrm{~m}$ long packed-column and $\mathrm{CO}_{2}$ at $23 \mathrm{MPa}$ and $45^{\circ} \mathrm{C}$ were recently published by Shariff et al. [41].

M'Hamdi et al. [9] observed that, using pure $\mathrm{CO}_{2}$, only partial resolution between non-aromatic fraction and monoaromatic hydrocarbons was obtained on diesel fuels even though ASTM requirements were easily reached between saturates and alkylated monoaromatic test-compounds (Fig. 7 and 8). They used a mixed mobile-phase $\mathrm{CO}_{2}-\mathrm{SF}_{6}$ [42] and demonstrated the possible extension of the ASTM D5186 method for the determination of all groups except olefins (as Schwartz and Brownlee showed that only partial resolution

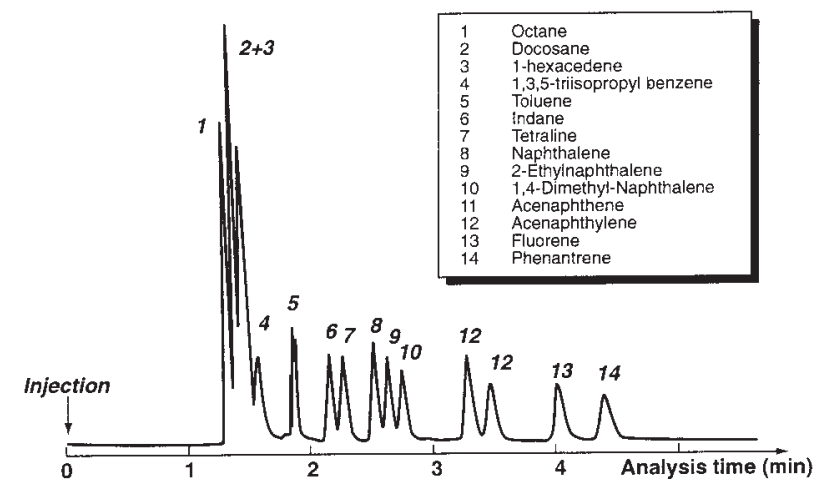

b)

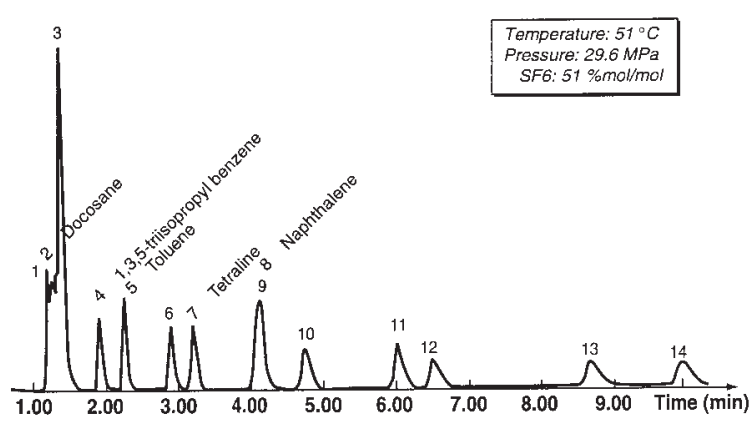

Figure 7. Chromatograms of a test mixture of hydrocarbons [42]. a) with pure $\mathrm{CO}_{2}$ at $30{ }^{\circ} \mathrm{C}$ and $15 \mathrm{MPa}$, b) with a $\mathrm{CO}_{2}-\mathrm{SF}_{6}$ mixture $(49 / 51 \% \mathrm{~mol} / \mathrm{mol})$ at $51{ }^{\circ} \mathrm{C}$ and $29.6 \mathrm{MPa}$. FID.

was obtained between olefines and saturates [43] using neat $\mathrm{SF}_{6}$ ). The addition of $\mathrm{SF}_{6}$ to the $\mathrm{CO}_{2}$ allows reduction of the solvent strength of the mobile phase leading to an increase of retention, selectivity and resolution. However, as pure $\mathrm{SF}_{6}$ did not enable elution of polyaromatic hydrocarbons, it was necessary to implement $\mathrm{CO}_{2}-\mathrm{SF}_{6}$ mixed mobile phases. Conventional LC columns were used and allowed independent control of the mobile-phase flow-rate and pressure via the pressure-regulation system of the chromatograph (downstream mode), while an integral restrictor was connected to the column outlet via a T-piece to split 1-2\% of the flow rate to the FID. The authors used an experimental design to define operating conditions, pressure, temperature and composition of the mobile phase, so the resolution between reference compounds was high, while the retention time was kept within 20 minutes. A mixture containing $51 \%$ (mol/mol) $\mathrm{SF}_{6}$ run at 29.6 $\mathrm{MPa}$ and $51{ }^{\circ} \mathrm{C}$ on a single hydrocarbon-group separation column (Hewlett-Packard, $250 \mathrm{~mm}$ $\times 4.6 \mathrm{~mm}$ ) leads to an increased resolution between saturates and mono-aromatics and between aromatics groups compared to the requirement of the ASTM method and to the HPLC IP391/95 method (Tab. III). Figure 7 compares the chromatograms obtained with pure $\mathrm{CO}_{2}$ and $\mathrm{CO}_{2}-\mathrm{SF}_{6}$ for a mixture of pure compounds and demontrates the resolution enhancement between the test compounds. Thus, better 
a)

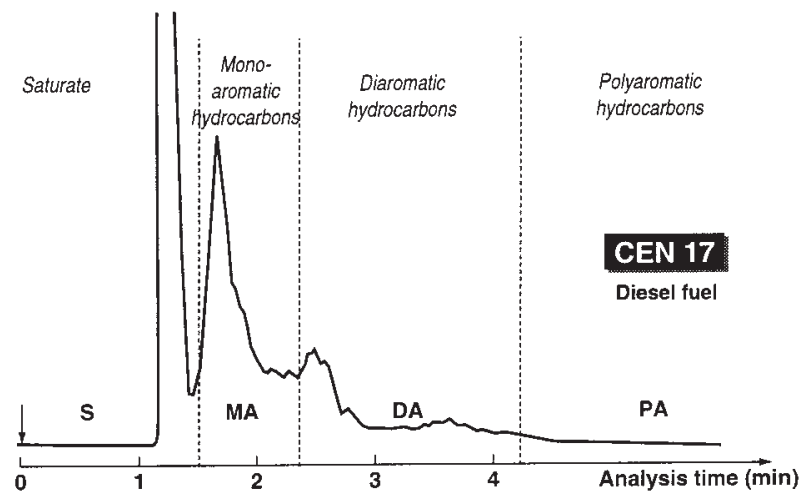

b)

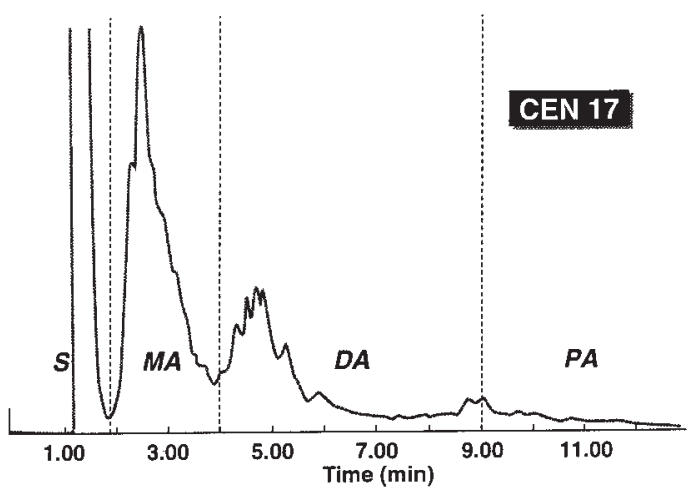

Figure 8. Chromatograms of the diesel fuel CEN17 [42]. a) with pure $\mathrm{CO}_{2}$, b) with a $\mathrm{CO}_{2}-\mathrm{SF}_{6}$ mixture. Same conditions as figure 7.

Table III. Requirements on resolutions for standardized methods and resolution achieved with $\mathrm{CO}_{2}-\mathrm{SF}_{6}$.

\begin{tabular}{lccc}
\hline Resolution & $\mathrm{D} / \mathrm{TBZ}(1)$ & $\mathrm{D} / \mathrm{TOL}(2)$ & $\mathrm{T} / \mathrm{N}(3)$ \\
\hline LC & - & $\geq 5.0$ & - \\
IP391/95 and \\
prEN12916
\end{tabular}

(1) Docosane 1 - 3,5-triisopropylbenzene.

(2) Docosane-toluene. Docosane is replaced by cyclohexane for the LC method and by hexadecane for the ASTM method. Toluene is replaced by o-xylene in the LC method.

(3) Tetraline-naphthalene. resolution of aromatic groups in diesel fuels is obtained as shown in figure 8. Compared to results reported by $\mathrm{Li}$ [40] using a long packed-capillary column $(52 \mathrm{~cm})$, the resolutions obtained using the mixed mobile-phase were higher, while the retention time of naphthalene was ca. 2 minutes shorter.

The method was evaluated using nine diesel fuels with an FBP up to $420{ }^{\circ} \mathrm{C}$ and a good correlation was obtained between SFC and MS results for the determination of aromatic groups [44]. This method could be further improved using a 2-mm ID column or even a microbore column to decrease $\mathrm{SF}_{6}$ consumption. Backflushing of di+ aromatics or pressure programming for an easier quantitation could also be included in the method.

\section{Crude oils: SARA method}

Skaar et al. [19] proposed an experimental procedure for SARA analysis involving a $1 \mathrm{~mm}$ silver-loaded cationexchange silica column, $\mathrm{CO}_{2}$ as the mobile phase and SFCFID. The system was reported to offer good stability for one crude oil analysis for two months. They used response factors of 1 for paraffins and aromatics and 0.7, 0.8 for resins and asphaltenes respectively from reported TLC-FID data using Iatroscan [45]. However, as resins and asphaltenes were backflushed as one single peak, quantitation of the four SARA fractions required a deasphalting step prior to SFC separation.

\section{Conclusion}

Despite the fact that SFC has many advantages over GC and $\mathrm{LC}$, it is in competition with both. Replacement of such techniques is difficult because they are mature and well established. Benefits gained by the use of a "new" technique have to be evidenced, and balanced with the costs and the time spent for switching from one method to another. From this point of view, SFC suffers from the limited number of instruments available, and the cost of integrated systems. Some systems have pressure limits too low for simdis application.

However, SFC has general features that merit wider applications based on the following benefits:

- short analysis and method-development times increase the sample number throughput;

- use of a "green" mobile phase reduces waste disposal cost while dissolving a wide range of molecules efficiently;

- availability of most of the detectors used in LC and GC offers the widest possibility of combining universal, informative and selective detections in chromatography.

For the petroleum applications, SFC is well adapted to the characterization of heavy hydrocarbons because of the solvating properties of supercritical fluids. SFC could be applied in routine simulated distillation for extending the 
range of the technique up to $\mathrm{C}_{140}$ (atmospheric and vacuum residues). Detection facilities of SFC could also be used for that application, such as the coupling of FID-UV for easy differenciation of aromatic and non-aromatic hydrocarbons. Group-type separations gained worldwide recognition via ASTM method release, and advantages of SFC over LC are demonstrated for, at least, the analysis of conventional diesel fuels. Again, the dual UV/FID detection mode [46] is no doubt a very promising alternative for achieving reproducible and versatile quantitation of saturates and olefins for a wide range of different samples. Commercial systems are perfectly suited to this application of SFC [47].

\section{References}

1. Wright, B. W.; Smith, R. D. Anal. Chem. 1989, 101, 111.

2. Chester, T. L.; Pinkston, J. D.; Raynie, D. E. Anal. Chem. 1998, 70, 301R.

3. Peaden, P. A. J. High Resol. Chromatogr. 1994, 17, 203.

4. Roberts, I. Supercritical-fluid chromatography, in Adlard, E. R., Ed.; Chromatography in the Petroleum Industry; Elsevier: NL, 1995, Chap 11.

5. Schwartz, M. M.; Brownlee, H. E.; Boduszynski, R. G.; Su, F. Anal Chem. 1987, 59, 1393.

6. Durand, J. P.; Bré, A.; Béboulène, J. J., Ducrozet, A., Carbonneaux, S. 19th Int. Symp. on Capillary Chromatography and Electrophoresis, Wintergreen, May 1997.

7. Raynie, D. E.; Markides, K. E.; Lee, M. L. J. Microcol. Sep. 1991, 3, 423.

8. Thiébaut, D.; Caude, M.; Rosset, R. Analusis 1987, 15, 528.

9. M'Hamdi, R.; Thiébaut, D.; Caude, M. J. High Resolut. Chromatogr. 1997, 20, 545.

10. Sotty, Ph.; Rocca, J. L.; Grand, C. 15th Int. Symp. on Capillary Chromatography, Riva del Garda, May 1993.

11. Bouigeon, Ch., Thiébaut, D., Caude, M. unpublished results, 1996.

12. Huynh, V. K.; Thiébaut, D.; Caude, M.; Robert, E., to be published.

13. Hewlett Packard, Application notes 228-169 and 228-176, 1992.

14. Kelemidou, K.; Severin, D. Erdöl Erdgas Kohle, 1996, 112, 25.

15. Shariff, S. M.; Tong, D.; Bartle, K. D. J. Chromatogr. Sci. 1994, 32, 541.

16. Schwartz, H. E. J. Chromatogr. Sci. 1988, 26, 275.

17. Berger, T. A. Packed-column SFC, RCS Chromatography Monographs; Smith, R. M Ed.; 1995.

18. Skaar, H.; Norli, H. R.; Lundanes, E.; Greibrook, T. J. Microcol. Sep. 1990, 2, 222.

19. Schoenmakers, P. J. 22 $2^{\text {nd }}$ International Symposium on Chromatography, Roma, 1998.
20. Greibrokk, T.; Lundanes, E.; Trones, R.; Molander, P.; Roed, L.; Skuland, I. L.; Andersen, T.; Bruheim I.; Jachwitz, B. In Unified Chromatography; Chester, T. and Parcher, J.; Ed.; ACS series: Washington, DC, submitted.

21. Molander, P.; Ommundsen E.; Greibrokk, T. J. Microcol. Sep. in press.

22. Robert, E. In Practical Supercritical-Fluid Chromatography and Extraction, Thiébaut, D. and Caude, M. Eds; Harwood Academic Publishers: U.K., 1999.

23. Burdett, R. A.; Taylor, L. W.; Jones, L. C. J. Molecular Spectroscopy, Rept. Conf., Inst. Petroleum: London, 1954, 30.

24. Trisciani, A.; Munari, F. J. High. Resolut. Chromatogr. \& Chromatogr. Comm. 1994, 17, 452.

25. Pal, R.; Juhasz, M.; Stumpf, A. J. Chromatogr. A 1998, 819A, 249.

26. Robert, E.; Béboulène, J. J.; Codet, G.; Enache, D. J. Chromatogr. A 1994, 683, 215.

27. Snyder, L. R.; Buell, B. E.; Howard, H. E. Anal. Chem. 1968, 40, 1303.

28. Radke, M.; Willsch, H.; Welte, D. H. Anal. Chem. 1980, 52, 406.

29. Lundanes, E.; Greibrokk, T. J. High Resolut. Chromatogr. 1994, 17, 197.

30. Di Sanzo, F. P.; Yoder, R. E. J. Chromatogr. Sci. 1991, 29, 4.

31. Fraile, R.; Sanchez, V. J. High Resolut. Chromatogr. 1993, 16, 169.

32. Hewlett Packard, Application notes 228-167, 1992.

33. Analytical Control, Application notes 9317-9318, 1993.

34. Norris, T. A.; Rawdon, M. G. Anal. Chem. 1984, 56, 1767.

35. Campbell, R. M.; Djordjevic, N. M.; Markides, K.; Lee, M. L. Anal. Chem. 1988, 60, 356.

36. Fuhr, B. J.; Klein, L. L.; Reichert, C.; Lee, S. W. LC-GC 1990, 8, 800 .

37. Anderson, P. E.; Demirbücker, M.; Blomberg, L. G. J. Chromatogr. Sci. 1992, 29, 4.

38. Anderson, P. E.; Demirbücker, M.; Blomberg, L. G. J. Chromatogr. 1993, 641, 347.

39. Chen, E. N.; Cusatis, P. D.; Popiel, E. J. J. Chromatogr. 1993, 637, 181.

40. Li, W.; Malik, A.; Lee, M. L.; Jones, B. A.; Porter, N. L.; Richter, B. E. Anal. Chem. 1995, 67, 647.

41. Shariff, S. M.; Robson, M. M.; Myers, P.; Bartle, K. D.; Clifford, A. A. Fuel 1998, 77, 927.

42. M'Hamdi, R.; Thiébaut, D.; Caude, M.; Robert, E.; Grand, C. J. High Resolut. Chromatogr. 1998, 21, 94.

43. Schwartz, H. E.; Brownlee, R. G. J. Chromatogr. 1986, 353, 77.

44. M’Hamdi, R.; Guénégou, T.; Thiébaut, D.; Caude, M.; Robert, E. in preparation.

45. Barhati, S.; Patience, R.; Mills, N.; Hanesand, T. Org. Geochem. 1997, 26, 49.

46. Shultz, W. W.; Genowitz, M. W. US Patent 5, 190, 882, 1993.

47. Richter, B. E.; Jones, B. A.; Porter, N. L. J. Chromatogr. Sci. 1998, 36, 444. 